\title{
Transmit Diversity and Equalization for Power Controlled Wireless Networks
}

\author{
Farrokh Rashid-Farrokhi, K.J. Ray Liu, and Leandros Tassiulas \\ Electrical Engineering Department and Institute for Systems Research \\ University of Maryland \\ College Park, MD 20742, USA
}

\begin{abstract}
A multitap transmit diversity scheme for the downlink is proposed where the transmit weight vectors are adjusted such that the SNR at each mobile is above a threshold. The proposed transmit diversity scheme can be applied to networks with multipath fading channels and in cases where the number of cochannels and multipaths are larger than the number of antenna elements.
\end{abstract}

\section{Introduction}

The capacity of a cellular system is limited by the cochannel interference (CCI) and Inter-Symbol Interference (ISI). CCI is due to the interference caused by users sharing the same channel. If the delay spread in a multipath channel is larger than a fraction of a symbol, the delayed components cause ISI. In the uplink, adaptive receiver beamforming schemes have been widely used to reduce the interference at the base station. In order to optimally reduce $\mathrm{CCI}$ and ISI, the space-time diversity combining has to be implemented jointly. Because of the large delay spread in the wireless channels, the joint space-time processing will improve the performance by reducing the $\mathrm{CCI}$ and ISI and increasing the SNR significantly [1]-[3].

Most often, deploying antenna arrays at the mobile is impractical. However, transmit diversity can be deployed at the base station to improve the downlink capacity. In scenarios where antenna arrays are used at transmitters, the beam-pattern of each antenna array can be adjusted to minimize the induced interference to undesired receivers. The transmit diversity and receiver beamforming are substantially different in nature. Receiver beamforming can be implemented independently at each receiver, without affecting the performance of other links, while transmit beamforming will change the interference to all other receivers. As a result transmit beamforming has to be done jointly in the

* This work is supported in part by the NSF grant MIP9457397, NSF grant NCR-9406415, CAREER award NCR-9502614. entire network. Moreover, in receiver beamforming a local feedback from the receiver output is used to adjust the combining vector. In transmit beamforming, the probing has to be done at the mobile, while the beampatterns are adjusted at the base stations [4]. In Time Division Duplex (TDD) systems where the uplink and downlink channels are reciprocal, the uplink channel information can be used for downlink [2], [5].

In [6] we proposed a distributed algorithm that jointly optimizes the mobile transmitted power and the uplink beamforming vector at a base station. In this work we propose an algorithm that finds a set of feasible space-time transmit diversity weight vectors and power allocations to achieve the required SNR at each link in the downlink.

\section{Transmit Space-Time Diversity}

Consider a set of $M$ cochannels where only transmitters are using antenna arrays with $P$ elements. In the following we will present an algorithm that finds a set of multitap diversity weight vectors for transmitters such that the desired SNR at each mobile is guaranteed. As we will see later, the transmitted power is also controlled by the transmit diversity weight vectors. The block diagram of a multitap transmit diversity system is shown in Fig. 1. The structure of a splitter is shown in Fig. 2. It should be noted that there is a dedicated multitap diversity system at the base station for each mobile.

The received signal at the $i$ th mobile is a superposition of the transmitted signal and its delayed versions, generated by the multipath channel. The transmitted vector by itself is a weighted combination of the desired signal and its delayed versions. Suppose at each link there are $L$ paths. Also assume that in the steady state, the weight vectors are time independent, and denote the diversity vector at the $q$ th tap of the $b$ th base station by $\mathbf{w}_{b}(q)$. With that as given and the slow fading assumption, the received signal at the $i$ th 
mobile is given by

$$
\begin{aligned}
y_{i}(t)=\sum_{b=1}^{B} & \sqrt{\rho_{i b} G_{i b} P_{b}} \sum_{q=0}^{Q-1} \sum_{l=1}^{L} \sqrt{\alpha_{i b}^{l}} \mathbf{w}_{b}^{H}(q) \mathbf{a}_{i b}\left(\theta_{l}\right) \\
& \times u_{i b}\left(t-\tau_{i b}^{l}-q T\right)+n_{i}(t),
\end{aligned}
$$

where $\rho_{i b}$ models the log-normal shadow fading, $\alpha_{i b}^{l}$ and $G_{i b}$ are the $l$ th path fading and loss from the $i$ th mobile to the $b$ th base station, respectively. $\tau_{i b}^{l}$ denotes the path delay. The length of equalizer is denoted by $Q . \mathbf{a}_{i b}\left(\theta_{l}\right)=$ $\left[a_{i b}^{1}\left(\theta_{l}\right) \ldots a_{i b}^{P}\left(\theta_{l}\right)\right]^{T}$ is the array response to the signal coming from the $i$ th mobile at direction $\theta_{l}$. Thermal noise at the $i$ th mobile is denoted by $n_{i}(t)$. The signal $u_{i b}(t)$ can be expressed as a function of the message symbols $s_{b}(n)$ by

$$
u_{i b}(t)=\sum_{n=0}^{k} g_{i b}(t-n T) s_{b}(n)
$$

where $k=\left\lfloor\frac{t}{T}\right\rfloor, T$ is the symbol interval, and $g_{i b}$ models the effect of waveform and channel response. We assume that a filter with the impulse response $f_{i}(t)$ is used at the $i$ th receiver whose output is sampled at symbol intervals $(t=$ $k T$ )

$$
\begin{aligned}
y_{i}(k) & =\sum_{b=1}^{B} \sum_{q=0}^{Q-1} \sum_{n=0}^{k} \sum_{l=1}^{L} \sqrt{\rho_{i b} G_{i b} P_{b}} \mathbf{w}_{b}^{H}(q) \mathbf{a}_{i b}\left(\theta_{l}\right) \\
& \times \sqrt{\alpha_{i b}^{l}} s_{b}(n) R_{i b}(k-n)+n_{i}(k),
\end{aligned}
$$

where

$$
\begin{aligned}
R_{i b}(k-n) & =\int g_{i b}\left(t-n T-\tau_{i b}^{l}-q T\right) f_{i}(t-k T) d t \\
& =\int g_{i b}\left(t-\tau_{i b}^{l}\right) f_{i}(t-(k-n-q) T) d t
\end{aligned}
$$

and

$$
n_{i}(k)=\int n_{i}(t) f_{i}(t-k T) d t .
$$

That is, the channel can be modeled by a discrete impulse response given by

$$
\mathbf{h}_{i b}(n)=\sum_{l=1}^{L} \mathbf{a}_{i b}\left(\theta_{l}\right) \sqrt{\rho_{i b} G_{i b} \alpha_{i b}^{l}} R_{i b}(n) .
$$

Assuming that the length of the discrete impulse response is $\mathrm{N}$, we can rewrite (1) as

$$
\begin{gathered}
y_{i}(k)=\sum_{b=1}^{B} \sum_{q=0}^{Q-1} \sum_{n=0}^{N-1} \mathbf{w}_{b}^{H}(q) \mathbf{h}_{i b}(n) \sqrt{P_{b}} s_{b}(k-n-q) \\
+n_{i}(k) .
\end{gathered}
$$

It follows that

$$
y_{i}(k)=\sum_{b=1}^{B} \sum_{q=0}^{Q-1} \mathbf{w}_{b}^{H}(q) \mathbf{H}_{i b} \sqrt{P_{b}} \mathbf{s}_{b}(k-q)+n_{i}(k),
$$

where

$$
\mathbf{H}_{i b}=\left[\begin{array}{llll}
\mathbf{h}_{i b}(0) & \mathbf{h}_{i b}(1) & \ldots & \mathbf{h}_{i b}(N-1)
\end{array}\right]
$$

and

$$
\mathbf{s}_{b}(k)=\left[\begin{array}{c}
s_{b}(k) \\
\vdots \\
s_{b}(k-N+1)
\end{array}\right] .
$$

Define

$$
\mathbf{W}_{b}=\left[\begin{array}{c}
\mathbf{w}_{b}(0) \\
\vdots \\
\mathbf{w}_{b}(Q-1)
\end{array}\right]
$$

and

$$
\mathbf{X}_{i b}(k)=\left[\begin{array}{c}
\mathbf{x}_{i b}(k) \\
\vdots \\
\mathbf{x}_{i b}(k-Q+1)
\end{array}\right],
$$

where $\mathbf{x}_{i b}(k)=\mathbf{H}_{i b} \sqrt{P_{b}} \mathrm{~s}_{b}(k)$. Then the received signal at the $i$ th receiver is expressed as

$$
y_{i}(k)=\sum_{b} \mathbf{W}_{b}^{H} \mathbf{X}_{i b}(k)+n_{i}(k) .
$$

We evaluate the SNR at each mobile as a function of the gain matrix $\mathbf{H}_{i b}$, weight vector $\mathbf{W}_{i}$, and transmitted powers $P_{b}$. The vector $\mathbf{X}_{i b}(k)$ is given by

$$
\mathbf{X}_{i b}(k)=\left[\begin{array}{c}
\mathbf{H}_{i b} \sqrt{P_{b}} \mathbf{s}_{b}(k) \\
\vdots \\
\mathbf{H}_{i b} \sqrt{P_{b}} \mathbf{s}_{b}(k-Q+1)
\end{array}\right] .
$$

We assume that the transmitted signals from different base stations are uncorrelated and zero mean. The total received power at the $i$ th mobile is then given by

$$
\mathcal{E}_{i}=E\left\{\left|y_{i}(k)\right|^{2}\right\}=\sum_{b} \mathbf{W}_{b}^{H} E\left\{\mathbf{X}_{i b}(k) \mathbf{X}_{i b}^{H}(k)\right\} \mathbf{W}_{b},
$$

Using (3), we rewrite the received power as

$$
\begin{gathered}
\mathcal{E}_{i}=\sum_{b=1}^{B} P_{b} \mathbf{W}_{b}^{H} E\left\{\left[\begin{array}{c}
\mathbf{H}_{i b} \mathbf{s}_{b}(k) \\
\vdots \\
\mathbf{H}_{i b} \mathbf{s}_{b}(k-Q+1)
\end{array}\right] \times\right. \\
{\left[\begin{array}{lll}
\mathbf{s}_{b}^{H}(k) \mathbf{H}_{i b}^{H} & \ldots & \left.\mathbf{s}_{b}^{H}(k-Q+1) \mathbf{H}_{i b}^{H}\right]
\end{array}\right] \mathbf{W}_{b}+N_{i},}
\end{gathered}
$$


where $N_{i}$ is the noise power at the $i$ th mobile. Suppose the signal transmitted from a source is also an uncorrelated zero mean sequence of symbols. Then (4) can be simplified as

$$
\mathcal{E}_{i}=\sum_{b=1}^{B} P_{b} \mathbf{W}_{b}^{H} \mathbf{G}_{i b} \mathbf{W}_{b}+N_{i}
$$

$\mathbf{G}_{i b}$ is a block matrix whose $p q$ th block defined by

$$
\left[\mathbf{G}_{i b}\right]_{p q}=\mathbf{H}_{i b} \mathbf{J}_{p, q} \mathbf{H}_{i b}^{H}
$$

where $\mathbf{J}_{p, q}$ is a matrix which only has ones on $(q-p)$ th diagonal in parallel with main diagonal elements. Assume we are estimating $s_{b}(k-Q / 2)$. The desired signal power is then given by

$$
P_{b} \mathbf{W}_{b}^{H} \mathbf{g}_{i i} \mathbf{g}_{i i}^{H} \mathbf{W}_{b}
$$

where

$$
\mathbf{g}_{i i}=\left[\begin{array}{c}
\mathbf{0} \\
\mathbf{h}_{i i}(N-1) \\
\vdots \\
\mathbf{h}_{i i}(0) \\
\mathbf{0}
\end{array}\right]
$$

The matrix $\mathbf{G}_{i i}$ can be separated into signal and interference matrices:

$$
\mathbf{G}_{i i}=\mathbf{G}_{i i}^{s}+\mathbf{G}_{i i}^{I},
$$

where $\mathbf{G}_{i i}^{s}=\mathbf{g}_{i i} \mathbf{g}_{i i}^{H}$. The power of the desired signal at the $i$ th mobile is given by $P_{i} \mathbf{W}_{i}{ }^{H} \mathbf{G}_{i i}^{s} \mathbf{W}_{i}$, and the interference power is $\sum P_{b} \mathbf{W}_{b}{ }^{H} \mathbf{G}_{i b}^{I} \mathbf{W}_{b}$, where

$$
\mathbf{G}_{i b}^{I}=\left\{\begin{array}{ll}
\mathbf{G}_{i b} & b \neq i \\
\mathbf{G}_{i i}-\mathbf{G}_{i i}^{s} & \text { otherwise }
\end{array} .\right.
$$

The signal to noise ratio at this receiver is given by

$$
\Gamma_{i}=\frac{P_{i} \mathbf{W}_{i}^{H} \mathbf{G}_{i i}^{s} \mathbf{W}_{i}}{\sum_{b} P_{b} \mathbf{W}_{b}^{H} \mathbf{G}_{i b}^{I} \mathbf{W}_{b}+N_{i}}
$$

We define the transmit diversity problem as to find the power allocation and weight vectors such that the link quality is satisfied at each link. That is,

$$
\Gamma_{i}=\gamma_{i}, \quad(i=1, \ldots, M)
$$

Consider a set of beamforming vectors $\mathbf{A}=\left\{\mathbf{W}_{1}\right.$, $\left.\ldots, \mathbf{W}_{B}\right\}$. A set of cochannel links is feasible if there exists a power vector $\mathbf{P}$, and a set $\mathbf{A}$ such that the link quality is satisfied for each link. For a fixed beamforming set $\mathbf{A}$ the power allocation is given by

$$
P_{i}=\gamma_{i}\left(\frac{\sum_{b} P_{b} \mathbf{W}_{b}^{H} \mathbf{G}_{i b}^{I} \mathbf{W}_{b}+N_{i}}{\mathbf{W}_{i}^{H} \mathbf{G}_{i i}^{s} \mathbf{W}_{i}}\right) .
$$

In matrix form the above equation can be expressed as

$$
\mathbf{P}=\mathbf{D}_{w} \mathbf{F}_{w}^{T} \mathbf{P}+\mathbf{u}
$$

where

$$
\left[\mathbf{F}_{w}\right]_{i j}=\mathbf{W}_{i}^{H} \mathbf{G}_{j i}^{I} \mathbf{W}_{i}
$$

and

$$
\mathbf{D}_{w}=\operatorname{diag}\left\{\frac{\gamma_{1}}{\mathbf{W}_{1}^{H} \mathbf{G}_{11}^{s} \mathbf{W}_{1}}, \ldots, \frac{\gamma_{M}}{\mathbf{W}_{M}^{H} \mathbf{G}_{M M}^{s} \mathbf{W}_{M}}\right\} .
$$

$\mathbf{u}_{w}$ is defined as

$$
\left[\mathbf{u}_{w}\right]_{i}=\frac{\gamma_{i} N_{i}}{\mathbf{W}_{i}^{H} \mathbf{G}_{i i}^{s} \mathbf{W}_{i}} .
$$

If the spectral radius of $\mathbf{D}_{w} \mathbf{F}_{w}^{T}$ is less than unity, the matrix $\mathbf{I}-\mathbf{D}_{w} \mathbf{F}_{w}^{T}$ is invertible and its inverse is positive. The solution to (8) is then given by

$$
\mathbf{P}=\left[\mathbf{I}-\mathbf{D}_{w} \mathbf{F}_{w}^{T}\right]^{-1} \mathbf{u} .
$$

The above solution can be achieved using an iterative algorithm where at each iteration the power vector is updated by

$$
\mathbf{P}^{n+1}=\mathbf{D}_{w} \mathbf{F}_{w}^{T} \mathbf{P}^{n}+\mathbf{u} .
$$

In the following we consider the problem of joint computation of a feasible set of combining weight vectors and power allocations. In order to achieve a feasible solution for downlink, we run the diversity combining for a virtual uplink network whose channel responses are similar to that of the downlink, and at each iteration we use the same combining vector for the downlink. The algorithm steps at the $n$th iteration are as follows:

\section{Algorithm:}

1. Diversity combining and equalization for virtual uplink:

$$
\mathbf{W}_{i}^{n}=\arg \min _{\mathbf{w}_{i}} \gamma_{i} \frac{\sum_{m} P_{m} \mathbf{W}_{i}^{H} \mathbf{G}_{i b}^{I} \mathbf{W}_{i}+N_{i} \mathbf{W}_{i}^{H} \mathbf{W}_{i}}{\mathbf{W}_{i}^{H} G_{i i}^{s} \mathbf{W}_{i}}
$$

2. Virtual uplink power update:

$$
\tilde{\mathbf{P}}^{n+1}=\mathbf{D}_{w}[n] \mathbf{F}_{w}[n] \mathbf{P}^{n}+\tilde{\mathbf{u}}_{w}[n],
$$

3. Downlink power update:

$$
\mathbf{P}^{n+1}=\mathbf{D}_{w}[n] \mathbf{F}_{w}^{T}[n] \mathbf{P}^{n}+\mathbf{u}_{w}[n]
$$

where

$$
\left[\mathbf{F}_{w}[n]\right]_{i j}=\left(\mathbf{W}_{i}^{n}\right)^{H} \mathbf{G}_{j i}^{I} \mathbf{W}_{i}^{n}
$$

and

$$
\mathbf{D}_{w}[n]=\operatorname{diag}\left\{\frac{\gamma_{1}}{\left(\mathbf{W}_{1}^{n}\right)^{H} \mathbf{G}_{11}^{s} \mathbf{W}_{1}^{n}}, \ldots, \frac{\gamma_{M}}{\left(\mathbf{W}_{M}^{n}\right)^{H} \mathbf{G}_{M M}^{s} \mathbf{W}_{M}^{n}}\right\}
$$

Positive vectors $\mathbf{u}_{w}$, and $\tilde{\mathbf{u}}_{w}$ are defined as 


$$
\left[\tilde{\mathbf{u}}_{w}[n]\right]_{i}=\frac{\gamma_{i} N_{i}\left(\mathbf{W}_{i}^{n}\right)^{H} \mathbf{W}_{i}^{n}}{\left(\mathbf{W}_{i}^{n}\right)^{H} \mathbf{G}_{i i}^{s} \mathbf{W}_{i}^{n}}
$$

and

$$
\left[\mathbf{u}_{w}[n]\right]_{i}=\frac{\gamma_{i} N_{i}}{\left(\mathbf{W}_{i}^{n}\right)^{H} \mathbf{G}_{i i}^{s} \mathbf{W}_{i}^{n}}
$$

The transmitted downlink power at the $b$ th transmitter is given by,

$$
\sum_{q=0}^{Q-1} P_{b}\left(\mathbf{w}_{b}^{n}(q)\right)^{H} \mathbf{w}_{b}^{n}(q)=P_{b}\left(\mathbf{W}_{b}^{n}\right)^{H} \mathbf{W}_{b}^{n}
$$

The first two steps of the above algorithm are similar to the uplink power update equations. We can show that if a set of cochannel links is feasible, there exists a set of optimal weight vectors and power allocations, such that the transmitted powers are minimal among all feasible solutions for the uplink [6]. In [7] we have shown that in a feasible network, the power vector $\tilde{\mathbf{P}}$ converges to a feasible power allocation $(\hat{\mathbf{P}})$. Assuming that the virtual uplink is feasible, we conclude the beamforming vectors are converging to a fixed space-time diversity vectors given by

$$
\hat{\mathbf{W}}_{i}=\arg \min _{\mathbf{w}_{i}} \gamma_{i} \frac{\sum_{m} \hat{P}_{m} \mathbf{W}_{i}^{H} \mathbf{G}_{i b}^{I} \mathbf{W}_{i}+N_{i} \mathbf{W}_{i}^{H} \mathbf{W}_{i}}{\mathbf{W}_{i}^{H} G_{i i}^{s} \mathbf{W}_{i}} .
$$

As a result $\mathbf{D}_{w}[n]$ and $\mathbf{F}_{w}[n]$ are also converging to constant matrices. Since the convergence is asymptotic, the system described in algorithm B is an asymptotically constant system. An asymptotically constant system is asymptotically stable if the gain matrix has all its eigenvalues inside the unit circle [8]. The feasibility of virtual uplink implies that the eigenvalues of the gain matrix $\left(\mathbf{D}_{w}[n] \mathbf{F}_{w}[n]\right)$ are inside the unit circle. The downlink gain matrix $\mathbf{D}_{w}[n] \mathbf{F}_{w}^{T}[n]$ is also converging to a fixed matrix, which has the same eigenvalues as that of the virtual uplink gain matrix [7]. Therefore the downlink is an asymptotically constant system with all its eigenvalues inside the unit circle, which implies that the downlink is also asymptotically stable. Since the feasibility of downlink and virtual uplink are the same, the algorithm will achieve a feasible solution for the downlink if there exists any.

\section{Simulation Results}

In order to evaluate the performance of our algorithm in cochannel interference reduction, a network with hexagonal cells is simulated. The path loss is proportional to $r^{-4}$, where $r$ is the distance between the mobile and base station. For each link, four paths with log-normal shadow fading and Rayleigh multipath fading are considered. The angle of arrival for each path is a uniform random variable in $[0,2 \pi]$.
A total of 100 mobiles, depicted by dots in Fig. 3, are distributed randomly throughout the network, and the base stations are placed at the center of each cell. Fig. 4 shows the total base station power at each iteration. Different configurations of the equalizer length $(Q)$ and the number of array elements (P) are considered. ( $P=1, Q=1)$ curve shows the case where we use omnidirectional antennas and standard power control [9]. ( $P=1, Q=4)$ curve shows the case where we use an equalizer with omnidirectional antenna at each base station. The solid curve shows that by using our algorithm and equalizers with length four and arrays with nine elements at base stations, we can significantly reduce the total transmitted power in the downlink.

The total transmitted power as a function of the number of users is shown in Fig. 5. We define the maximum capacity of the network as the maximum number of users for which the total transmitter power is below a certain limit. The maximum capacity of the network is improved significantly as we increase the number of array elements and the length of the equalizer.

\section{Conclusion}

We have proposed an algorithm for transmit space-time diversity jointly with power control, which achieves a feasible set of space-time vectors and power allocations if there exists any. The proposed algorithm guarantees the required SNR at each link. We have shown that when we use adaptive arrays at the base station, using our algorithm, we can increase the downlink capacity many folds.

\section{References}

[1] P. Balaban, and J. Sulz, "Optimum combining and equalization in digital data transmission with application to cellular mobile radio," IEEE Trans. Vehich. Tech., May 1991, pp. 342-354..

[2] H. Liu and G. Xu, "Multiuser Blind Channel Estimation and Spatial Channel Pre-Equalization," In Proc. ICASSP'95", pp. 1756-1759, Detroit, MI, May 1995.

[3] E. Periha, G. Pottie, “Adaptive Antenna Arrays and Equalization for Indoor Digital Radio", IEEE Int. Conf. Comm. ICC-96, June 1996.

[4] D. Gerlach, and A. Paulraj, "Adaptive transmitting antenna array with feedback," IEEE Sig. Proc. Lett., vol. 1, No. 10, Oct. 1994.

[5] G. G. Raleigh, S. D. Diggavi, V. K. Jones, and A. Paulraj, "A blind adaptive transmit antenna algorithm for wireless communications," Proc. ICC'95, p. 1949 , vol.3, 1995. 
[6] F. Rashid-Farrokhi, L. Tassiulas, K.J.R. Liu, "Joint optimal power control and beamforming for wireless networks with antenna arrays," Proc. IEEE Global Communications Conference (GLOBECOM-96), pp. I-555-559, London, Nov. 1996.

[7] F. Rashid-Farrokhi, K.J.R. Liu, L. Tassiulas, “ Transmit and Receive Space-Time Diversity in Wireless Networks with Fading Channels," ISR technical report, Institute for System Research, University of Maryland at College Park, Nov. 1997.

[8] J. L. Willems, "Stability Theory of Dynamical Systems," John Wiley \& Sons, Inc., New York, 1970.

[9] J. Zander, "Distributed cochannel interference control in cellular radio systems," IEEE Trans. Vehicular Technol., vol. 41, Aug. 1992.

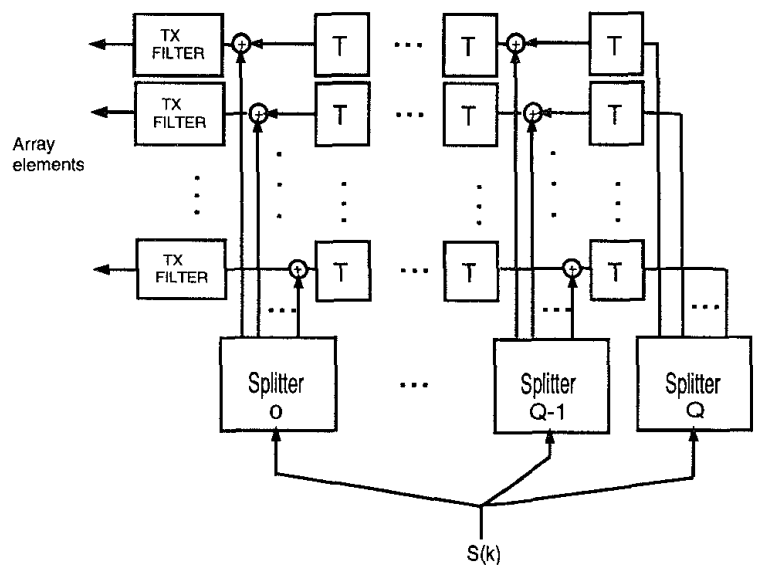

Figure 1. Block diagram of the transmit diversity system.

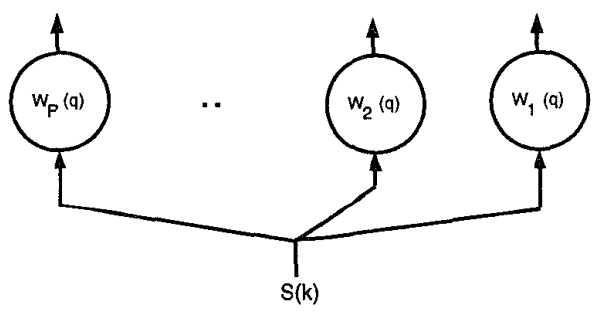

Figure 2. Power Splitter.

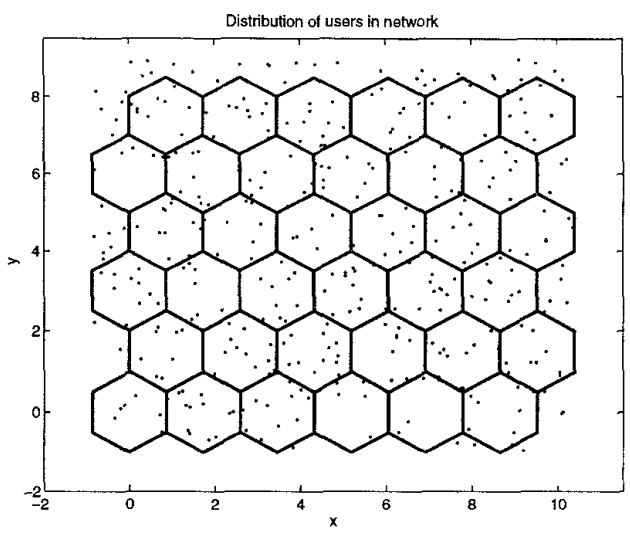

Figure 3. Mobile locations for 100 users.

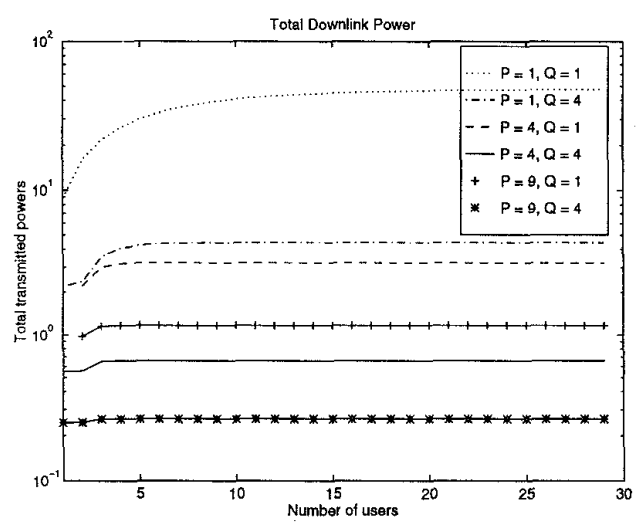

Figure 4. The total base station power at each iteration; 100 users and $\gamma=.03$; ( $\mathbf{P}$ : number of array elements, $Q$ : the length of equalizer).

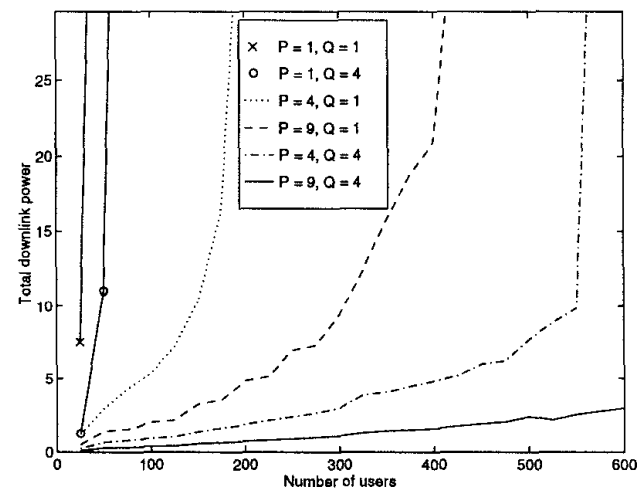

Figure 5. The total base station power as a function of the number of users; $\gamma=.05$. 